\title{
Determination of Ellagic Acid Compound Activity as Anti Breast Cancer
}

\author{
I Gusti Made Sanjaya* \\ Department of Chemistry \\ Universitas Negeri Surabaya \\ Surabaya, Indonesia \\ igmasanjaya@unesa.ac.id
}

\author{
Defitiana Wanita \\ Department of Chemistry \\ Universitas Negeri Surabaya \\ Surabaya, Indonesia \\ defitianawanita@mhs.unesa.ac.id
}

\begin{abstract}
This research was conducted to determine the activity of ellagic acid compounds as anti-breast cancer. The research was carried out through Molecular Docking in silico with several stages such as optimization of the structure of $3 D$ ellagic acid compounds and docking between optimized ellagic acid compounds and NUDT5 protein. Molecular docking is done using the software of Autodock Tools 1.5.6 and Discovery Studio 2016. The results showed that ellagic acid has the potential as an anti-breast cancer compound based on its binding energy to the NUDT5 protein of $\mathbf{- 3 . 9 8} \mathrm{kcal} / \mathbf{m o l}$ and an inhibitor constant of $1.21 \mathrm{mM}$.
\end{abstract}

Keywords—ellagic acid, molecular docking, NUDT5, binding energy, inhibitors constant

\section{INTRODUCTION}

Ellagic acid is an example of bioactive compounds found in various fruits such as cranberries, strawberries, raspberries, pomegranates. This compound is also found in a variety of nuts such as pecans and walnuts and in various vegetables [1]. Ellagic acid in various plants is found as ellagitannin [2]. An ellagic acid that is bound to a sugar molecule.

Ellagic acid is widely studied as a medicinal ingredient such as in the treatment of cancer [3], protection against brain injury in infants [1], improvement of cardiovascular function [4], and treatment of hyperpigmentation of the skin [5] [6]. This compound which is a heterocyclic compound is a dimerization product of gallic acid and has potential as an antioxidant [7] and antiproliferative [8]. Several studies have identified ellagic acid as being anticarcinogenic and antimutagenic [9].

This study examines the potential of ellagic acid as an anti-cancer. This is done considering the disease is degenerative and many victims. The number of cancer sufferers is increasing every year.

The most common types of cancer in society today are breast, liver, lymphoma, blood and cervical cancer. Breast cancer is the second most common cancer that causes death after lung cancer and is the most common cancer in women. According to WHO more than 1.2 million people were diagnosed with breast cancer [10].

Breast cancer is a disease in which malignant cells form in breast tissue. Breast cancer is commonly found in women, about 100 times more than breast cancer in men. Breast cancer sufferers in the world are relatively high [11]. According to 2012 GLOBOCAN data, it is known that breast cancer is cancer with the highest percentage of new cases in women, which is $43.3 \%$. Percentage of deaths from breast cancer by $12.9 \%$ [10].

The key process associated with breast cancer is NUDT5 (NUDIX hydrolase 5 or NUDIX5). There are two dominant substrates that have been identified for NUDT5, namely 8oxo-dGDP and adenosine $5^{\prime}$ diphosphoribose (ADPR). NUDT5 has evidence of hydrolyzing 8-oxo-dGTP under basic conditions $(\mathrm{pH} \approx 10)$. The physiological role of NUDT5 in the metabolism of 8-oxo-guanine has not been thoroughly studied. NUDT5 has recently been proven responsible for producing nuclear ATP mediated by PAR (protease-activated receptor). This results in chromatin remodelling that is dependent on ATP and gene regulation after stimulation of progestin or estrogen in breast cancer cells [12].

The research on anticancer compounds was conducted to determine the activity of ellagic acid compounds as anticancer compounds against NUDT5 protein using molecular docking methods. Where is expected ellagic acid compounds can inhibit the activity of breast cancer cells.

\section{MATERIALS AND METHODS}

The material used in this study is the $3 \mathrm{D}$ molecular structure of ellagic acid compounds which functions as a ligand and the 3D molecular structure of the NUDT5 protein. The two compounds were obtained from protein data banks.

Early molecular modeling uses Avogadro 1.2.0 software that works on the Microsoft Windows 10.1 operating system. The stability of molecular geometry is ensured through computational chemistry using software of Games. Autodock Tools 1.5.6 software is used to determine the position of a ligand that matches its receptor and is used to calculate the lowest affinity value in determining parameters in molecular docking. The molecular docking results are then displayed using Discovery Studio 216 software.

\section{RESULT AND DISCUSSION}

Visualization of ellagic acid carried out using Avogadro 1.2.0 software is shown in Figure 1 The ellagic acid file is then saved in the pdb file format. 


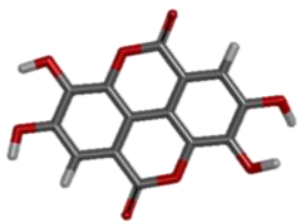

Fig. 1. Visualization of ellagic acid compounds in the pdb file format

This visualized ellagic acid compound is prepared as a ligand in the molecular docking process. The visualization of electron density from the computational results of ellagic acid compounds is shown in the following figure.

\section{Dipole = 1.196863 Debye}

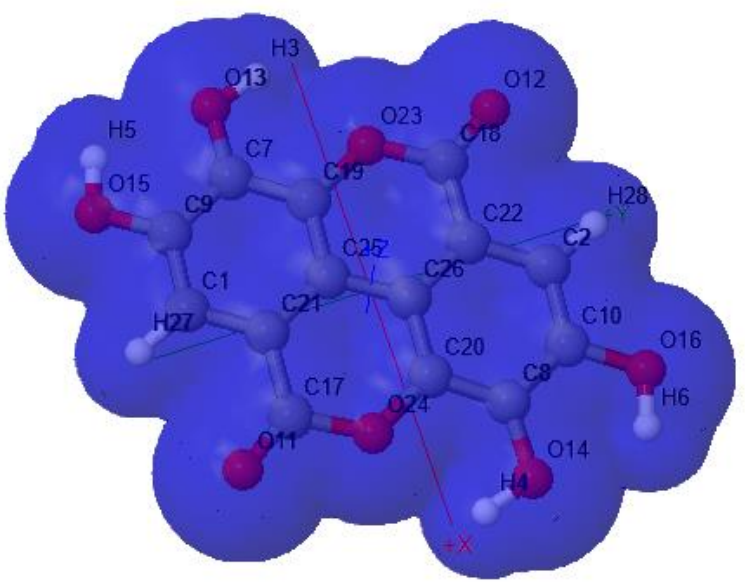

$\begin{array}{lllllllllll}0.20 & -0.16 & -0.12 & -0.08 & -0.04 & 0.00 & 0.04 & 0.08 & 0.12 & 0.16\end{array}$

Fig. 2. Visualization of the electron density of ellagic acid

These results indicate that elagic acid is a polar compound with a dipole moment of 1.196863 Debye. Based on this, it can be predicted that the ellagic acid molecular compounds are relatively stable and easily soluble in water. This is consistent with the physical properties data shown through the site https://pubchem.ncbi.nlm.nih.gov/ compound/Ellagic-acid\#section=Solubility. The results of the optimization of the ellagic acid molecular structure used as a ligand in the molecular docking are then stored with Discovery Studio 216 software in pdbqt format.

The target protein used in this study is the NUDT5 protein. This protein is the result of visualization of x-ray crystallography and Nuclear Magnetic Resonance (NMR) spectroscopy whose files are stored on the website www.pdb.org with the code 2DSB 5NQR. The NUDT5 protein file is downloaded and prepared using Autodock Tools to remove $\mathrm{H} 2 \mathrm{O}$ molecules and ligands from the protein. The results are shown in Figure 3

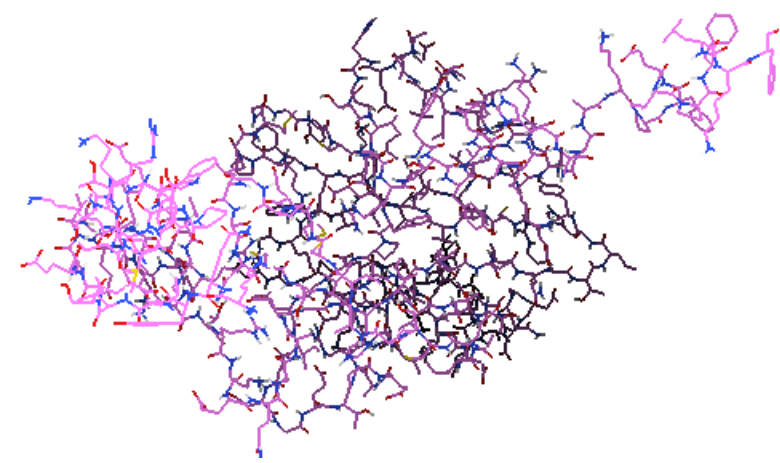

Fig. 3. Results of NUDT5 protein structure preparation

The 3D structure of the ellagic acid compound and the optimized NUDT5 protein were calculated for their lowest affinity value by determining parameters in molecular docking using the Autodocok Tools software. The coordinates of the ligand and protein interactions for the docking process are $\mathrm{X}, \mathrm{Y}, \mathrm{Z}=60$. Furthermore, then determining the dimensions of the grid box, the dimensions of the grid box $X=6.546, Y=39.984, Z=37.548$. The results of ligand-protein docking of ellagic acid-NUDT5 using Discovery studio 216 software are shown in Figure 3

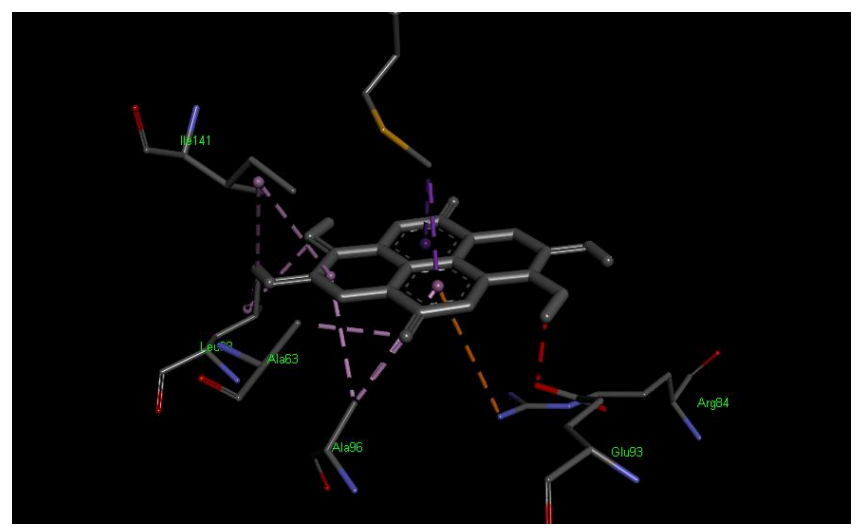

Fig. 4. docking molecule of ellagic acid compounds with NUDT5 protein

Figure 3 shows the interaction between the molecular ligands of ellagic acid compounds and the active sites of NUDT5. Interactions that occur involve hydrogen bonds between ellagic acid and NUDT5 residues, namely: Ile141; Leu98; Ala63; Ala96; Met132; Glu93; and Arg84. The interaction produces complex binding energy of -3.98 $\mathrm{kcal} / \mathrm{mol}$.

The negative energy sign indicates that the ligandbinding interaction that occurs on the active side of NUDT5 tends to be strong. This is an important aspect that must be considered. The increasingly negative binding energy indicates a compound requires less energy to bind. In other words, the process of interaction has become easier. The potential for ligand binding to the target protein is greater. This also shows the potential of ellagic acid to be an antibreast cancer compound. This potential is supported by an inhibitor constant value of $1.21 \mathrm{mM}$. The inhibitor constant (Ki), which is an indication of the inhibitory strength of the 
substrate and inhibitor in binding enzymes whose value is getting smaller, shows the greater binding affinity to inhibit the activity of the enzyme.

\section{CONCLUSIONS}

Molecular docking results showed that the interaction between ellagic acid molecular ligands and NUDT5 protein had a binding energy of $-3.98 \mathrm{kcal} / \mathrm{mol}$ and a $\mathrm{Ki}$ inhibitor constant of $1.21 \mathrm{mM}$. This shows that ellagic acid has the potential as anti-breast cancer that is able to attack the active sides of the NUDT5 protein consisting of Ile141; Leu98; Ala63; Ala96; Met132; Glu93; and Arg84.

\section{REFERENCES}

[1] U. E. Hernawan and A. D. Setyawan, "REVIEW: Ellagitannin; biosynthesis, isolation,," Biofarmasi, vol. 1, no. 1, pp. 25-38, February 2003

[2] S. -Y. Chen, K. Zheng and Z. -q. Wang, "Neuroprotective effects of Ellagic acid on Neonatal Hypoxic Brain Injury via Inhibition of Inflammatory Mediators and Down-regulation of JNK/p38 MAPK Activation," Tropical Journal of Pharmaceutical Research, vol. 15, no. 2, pp. 241-251, February 2016.

[3] Y. Wang, F. Ren, B. Li, Z. Song, P. Chen and L. Ouyang, "Ellagic acid exerts antitumor effects," J Cancer, vol. 10, no. 15, pp. 3303 3314, 2019.

[4] K. H. Nejad, M. Dianat, A. Sarkaki, M. K. Naseri, . M. Badavi and . Y. Farbood, "Ellagic acid improves electrocardiogram waves and blood pressure against global cerebral ischemia rat experimental models," Electron Physician, vol. 7, no. 4, p. 1153-1162, 10 Aug 2015.

[5] C. Couteau and L. Coiffard, "Review Overview of Skin Whitening
Agents: Drugs and Cosmetic," Cosmetics, vol. 3, no. 27, pp. 1-16, 2016.

[6] J. P. Ortonne and D. L. Bissett, " Review Latest Insights into Skin Hyperpigmentation," Journal of, vol. 13, pp. 10-14, 2008

[7] Y. -q. SUN, X. TAO, X. -m. MEN, Z. -w. XU and T. WANG, " In vitro and in vivo antioxidant," Journal of Integrative Agriculture, vol. 16, no. 8, pp. 1808-1818, Aug 2017.

[8] H. -A. Kim, R. -A. Lee, B. -I. Moon and K. -J. Choe, "Ellagic Acid Shows Different Antiproliferative Effects Between the MDA-MB-231 and MCF-7 Human Breast Cancer Cell Lines," J. Breast Cancer, vol. 12, no. 2, pp. 85-91, June 2009.

[9] G. Cozza, " The Development of CK2 Inhibitors: From Traditional Pharmacology to in," Pharmaceuticals (Basel), vol. 10, no. 1, p. 26, March 2017.

[10] Grobocan-IARC, Estimated Cancer Incidence, Mortality and Prevalence Worldwide in 212., Grobocan: World Health Organiation, 2012.

[11] J. Ferlay, H. Shin, B. Bray, F. Mathers and D. Parkin, Cancer Incidence and Mortality Worldwide: IARC Cancer Base No. 1. International agency for Research on Cancer, Grobocan: lyon, 2010.

[12] D. Brent and C. Nicholas, "Targeted NUDT5 Inhibitor Block Hormone Signaling in Breast Cancer Cells," J. Nature Communication, vol. 9, no. 250, 2018. 